\title{
Complications of minimally invasive left Ventricular assistance: a case report and review of the literature
}

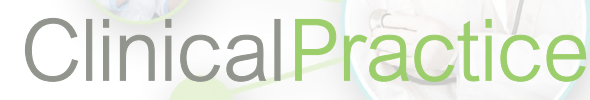

Ventricular assistance concerns patients with advanced cardiac failure in whom maximal medical and surgical treatment has been used. The ventricular assistance has two main purposes: first, to maintain circulation by discharging the ventricle (s) to provide recovery, and second to ensure patient survival by replacing cardiac function permanently or transitionally for patients waiting for cardiac Transplantation.

The encouraging results of the partial or total artificial heart and the miniaturization of these devices allow their use in permanent implantation for patients with cardiac insufficiency that is not eligible for transplantation.

In left mono-ventricular assistance, blood is taken from a cannula placed at the apex of the left ventricle (LV) and returned to the patient by an anastomosed vascular prosthesis with the ascending aorta. The classic surgical approach is a total median sternotomy. Other minimally invasive approaches for the implantation or explanation of left ventricular assist devices have been published and have shown encouraging results. These alternatives currently play an important role in certain indications and in patients with heavy medical history. Nevertheless, the complications of the ventricular assistance even by minimally invasive approaches might be serious and represent a turning point in the life of the patients.

We describe the implantation of a left ventricular assistance using HeartMate II device by left under costal and high mini sternotomy approach in a tridux patient with permeable coronary bypasses and we discuss its postoperative evolution.

\section{KEYWORDS: cardiac failure, hypertension, echocardiography, cardiac transplantation, hemorrhage}

\section{Case report}

Our case is about a 64 year-old man, with medical history of hypertension, diabetes and active smoking along with sleep apnea syndrome and coronary heredity. He has been followed for ischemic heart disease for which he was operated on twice: the first time in 1986 with surgical revascularization by 3 Coronary Artery Bypass Grafting (CABG) using only venous grafts and the second time in 2003 by a five bypasses using: the Left internal mammary artery on the LIVA and the diagonal along with 3 venous grafts on the left marginal, the right coronary and the right marginal [1,2]. Moreover, among the extra cardiac medical history our patient has hypothyroidism and had an ischemic stroke without neurologic deficiency.

The patient has been followed since 2008 for heart failure with permeable CABG. A multisite defibrillator was implanted in 2012 . He was operated in 2013 for renal adenocarcinoma without postoperative complications, and a good carcinological prognosis. For this, he was temporarily removed from the list of heart transplant.

Based on the increase in dyspnea stage (IIIBIV) with the need for a higher doses of diuretics, the necessity of introducing inotropes, and the echocardiography $\quad(\mathrm{LVEF}=20 \%, \quad \mathrm{RVEF}=40 \%$ and TAPSE $=15$ ) and catheterism data (a cardiac index at $1.8 \mathrm{l} / \mathrm{min} / \mathrm{m}^{2}$, PAP at $" 61 / 35 \mathrm{~mm} \mathrm{Hg}$ and Pcap P at $20 \mathrm{~mm} \mathrm{Hg}$ ); A collegial decision of a long-term circulatory assistance was taken.

A Thoratec HeartMate II device was implanted via a minimally invasive approach. The main arguments in favor of this approach were the presence of intrapericardial adhesions (Tridux) with permeable grafts. In addition, this young patient still a potential candidate for a cardiac transplantion, if a sufficient and satisfactory carcinological follow-up could be observed.

\section{Mleyhi Sobhi ${ }^{* 1}$ Radu Costin \& \\ Couetil Jean Paul}

Department of Cardiac Surgery, $\mathrm{CHU}$ Henri Mondor, University of Medicine Pierre et Marie Curie, Paris

*Author for correspondence: mleyhisobhi@yahoo.com 
The patient underwent:

- A "J" ministernotomy at the level of the manubrium up to the second right intercostal space to access the ascending aorta above the venous aortocoronary graft implantation site in order to be able to anastomose the Dacron prosthesis which represents the ejection path of the assistance device. Through the same incision an opening of the right pleura was done for the tunneling of the prosthesis.

- A second incision left undercostal of 10 $\mathrm{cm}$ with opening of the left pleura and the pericardium. This path allowed a satisfactory access to the apex of the heart and the creation of a place for the assistance device behind the rectus muscle as well as a tunnel to the right pleura.

To work safely and to avoid the risks of bleeding and cardiac arrest; A femoro-femoral extracorporeal circulation (ECC) was installed. The device inlet cannula was introduced and attached in the left ventricular apex. After the anterograde purging and tunneling of the prosthesis, a termino-lateral anastomosis between the prosthesis and the ascending aorta was performed [3]. The machine was put at $6000 \mathrm{rpm}$, then an anterograde purging under Trans-esophageal echocardiography (TEE) control was performed and finally the clamp on the prosthesis was removed. A progressive withdrawal of ECC with a concomitant increase in pump flow was achieved while maintaining a satisfactory hemodynamic status under 5 $\mathrm{\nabla g} / \mathrm{kg} / \mathrm{min}$ of noradrenalin and $0.8 \mathrm{mg} / \mathrm{h}$ of dobutamin. The duration of the ECC was 127 min and the patient was transfused with blood and platelets.

Immediate surgical follow-up was marked by bleeding requiring more transfusion with favorable evolution. The TEE control revealed the appearance of a thrombus in the noncoronary valsava sinus, a laminar flow without obstacle to the aspiration and a good LV discharge. The curative anticoagulation is started at $\mathrm{D} 1$ with an anti-Xa at 0.32 . The occurrence of heparin-induced thrombocytopenia urged us to relay by the Orgaran.

At D2 post-operative, the patient suddenly presented right hemiplegia and aphasia. A CT Scan was performed and did not find any embolus on the major arteries to the cerebral destination. The Transthoracic echocardiography (TTE) no longer finds the thrombus image of the noncoronary sinus. Moreover, there is a complete opening of the aortic valve at each cardiac cycle and the "ramp test" with progressive increase of the pump speed does not improve the left ventricular discharge, which suggests a thrombotic obstruction of the pump. The patient was urgently re-operated for changing of assistance device by the same approach. Once under femor-femoral ECC, the ejection path was clamped and the inlet cannula was removed. The inspection revealed a thrombus into the device, whereas the ventricular cavity was free from thrombi. A retrograde purge of the Dacron prosthesis was made and another Thoratec heratMate II device was placed.

A cerebral CT scan control after 2 days of the stroke showed voluminous gaps in the left internal and parieto-occipital capsule.

The short and medium term evolution was marked by the occurrence of several complications spread over a period of 8 months:

- Acute renal failure with progressive improvement.

- 3 re-interventions for bleeding of the box of the device and the right pleura; the last one by video-thoracoscopy at 1 month of the HeratMate implantation.

- Erysipelas treated with vancomycin for 7 days.

- Sliding syndrome with anxiety of death for which he was followed in psychiatry.

- Post-traumatic spleen fracture with hemoperitoneum requiring splenic truncal embolization.

- Discovery of multiple aneurysms of hepatic arteries probably of infectious origin requiring also embolization.

On the neurological level, the evolution was favorable with resumption of movement of the lower limb and of the walking but he maintains a paresis of the right upper limb. The anticoagulation is ensured by Previscan with a target INR between 1.8 and 2.2.

In October 2014, the patient presented with a right temporal hemorrhagic stroke with peri-lesional edema. The evolution was unfavorable with deterioration of his neurologic state concomitant with the increase in the size of the cerebral haemorrhage as well as the detection of the mycotic aneurysms in the cerebral angiography probably in relation with the infection of the pump. In view of this clinical and scannographic data of dark prognosis, a therapeutic limitation was decided 
and the patient was dead after 8 months of the implantation of the assistance.

\section{Discussion}

The impact of heart failure on individuals and society in general continues to grow. Cardiac transplantation remains the reference therapy to treat patients with end-stage cardiac failure. The development of partial and total artificial hearts was mainly inspired by the disproportion between the number of hearts available and the number of candidates for the transplantation. The important technological advances, made since the first studies of Kolff in 1957, offer more time to these patients awaiting the transplantation. The encouraging results of the artificial heart and the miniaturization of these devices now make it possible to consider their use in definitive implantation for patients with heart failure who are not eligible for transplantation [4].

The aims can be divided into two categories:

- Support and optimization of hemodynamic statue while attending the replacement of an irreversibly damaged heart (bridge-totransplant). This attitude prevents pulmonary hypertension and multiorganic failure; $80 \%$ of patients treated in this way have an evolution after transplantation equivalent to or better than the mean [5].

- Definitive implantation of a mechanical heart because the patient not a candidate for transplantation (destination therapy) has a 1 -year mortality greater than $50 \%$; Survival depends on the technical characteristics of the system and their intercurrent complications. It is currently $60-86 \%$ at 1 year, more than the double of maximum medical treatment [6-9].

Established early in the course of the disease, ventricular assistance can recover renal and hepatic function, reduce pulmonary hypertension, mobilize excess interstitial fluid and prevent Multiorganic failure. The criteria for implantation are the persistence of the following elements despite a maximum pharmacological treatment:

- Cardiac Index $<2.0 \mathrm{~L} / \mathrm{min} / \mathrm{m}^{2}$,

- $\mathrm{MAP}<60 \mathrm{mmHg}, \mathrm{SAP}<80 \mathrm{mmHg}$

- CVP and or OPAP>20 mmHg,

- $\mathrm{SAR}>2,000$ dynes $\bullet \mathrm{s} \bullet \mathrm{cm}^{5}$,

- $\mathrm{LVEF}<25 \%$, $\mathrm{VSO} 2<55 \%$,

- Urinary flow $<20 \mathrm{ml} /$ hour;

- Persistent metabolic acidosis.
Assistance should not be indicated in patients with active systemic infection, irreversible neurological deficiencies, and end-stage renal or hepatic impairment [10], also in aortic valve regurgitation; otherwise the flow of assistance returns back into the $\mathrm{LV}$ which becomes dilated.

With severe pulmonary hypertension or right heart failure; bi-ventricular assistance (20\% of cases) is required.

In our patient, the indication for implantation of long-term left ventricular assistance was based on the young age of the patient, dyspnea stage IIIB of NYHA with a cardiac index at $1.8 \mathrm{l} /$ $\mathrm{min} / \mathrm{m}^{2}$ despite an optimal medical treatment for 2 months and the presence of a temporary contraindication to cardiac transplantation (adenocarcinoma of the right kidney).

Certainly ventricular assistance is an expensive therapy. Pumps implanted in the long term (destination therapy) costs about $220,000 €$ [11]. Ventricular assist systems can be classified in different kinds. Historically, there are three generations $[1,12]$.

- The 1 st generation: extracorporeal pulsatil devices, often pneumatic, driven by an external console (Thoratec PVAD ${ }^{\mathrm{TM}}$, Abiomed BVS $\left.5000^{\mathrm{TM}}\right)$; they are bulky and contain many moving parts, including valves.

- The 2nd generation: implantable pulsatil systems, mostly electrical (HeartMate XVE ${ }^{\mathrm{Tm}}$, LionHeart $^{\mathrm{TM}}$, Thoratec IVAD $^{\mathrm{TM}}$ ).

- The 3rd generation: implantable axial flow system, for long durations (HeartMateII ${ }^{\mathrm{TM}}$, Jarvik $2000^{\text {Tm }}$, BerlinHeart $\left.{ }^{\mathrm{TM}}\right)$; The only moving part is the rotor, the valves are useless; The last models operate by magnetic system which removes the axes of the rotor, sources of wear.

We have opted for the implantation of a "HEARTMATE II" device in this patient due to the presence among his risk factors of a hypertension and a stroke. It is an electrical and intra-corporeal circulatory assistance device with continuous flow for the left ventricle. This system is indicated when the body surface area of the patient is $\geq 1.2 \mathrm{~m}^{2}$ in the following situations:

- Indication in acute situation: acute mono or bi ventricular failure in cardiac insufficiency, not controlled by an optimal medical treatment, in the absence of conventional therapeutic (pharmacological and/or interventional and/ or surgical) alternative. 
- Elective indication: advanced chronic heart failure with mono- or bi-ventricular failure, when life is threatened despite optimal medical treatment, and after a multidisciplinary decision. This is the case of our patient.

Contraindications to HEARTMATE II are:

- Severe pulmonary dysfunction and fixed pulmonary arterial hypertension.

- Severe hepatic insufficiency (cirrhosis, portal hypertension...).

- Major blood vessel disorders and uncontrolled bleeding.

- Uncontrolled systemic septic and inflammatory syndrome.

- Documented irreversible central nervous system lesions, recent stroke and cachexia.

- Systemic diseases interesting multiple organs.

- Psychiatric disorders with lack of cooperation.

- A serious disease with life expectancy less than 2 years.

- Body surface area $<1.2 \mathrm{~m}^{2}$.

"HeartMate II" is part of continuous flow systems that are driven by an axial turbine (HeartMate $^{\mathrm{TM}}$ II, BerlinHeart ${ }^{\mathrm{TM}}$, Jarvik 2000 ${ }^{\mathrm{TM}}$, DeBakeyMicroMed $^{\mathrm{TM}}$ ) or a centrifugal pump (HeartMate $^{\mathrm{TM}}$ III). They are implanted by sternotomy or by thoracotomy. The blood is taken at the apex of the LV and returned to the ascending or descending aorta by a tubular prosthesis. The flow rate is 3 to $10 \mathrm{~L} / \mathrm{min}$. These simpler and quieter continuous flow systems are less prone to embolism and infection than pulsatil systems [12,13]. In addition, they let the heart continue to eject which promotes its recovery. In another hand, the device is smaller, so it can be implanted in patients of small size.

The smaller pump design and its intrapericardial location allowed the development of less invasive implantation alternatives. The minimally invasive approach has been described in several publications [3,14-17]. It consists of a superior J-sternotomy or anterior thoracotomy in the second right intercostal space for access to the ascending aorta (anastomosis site of the ejection prosthesis) in addition to the right atrium if an atrio-cave Cannulation was needed and left under costal or left antero-lateral thoracotomy approach of $8-10 \mathrm{~cm}$ for access to the apex of the heart and implantation of the device.

According to Anson Cheung et al. [16], the use of small incisions allows exposure of the exact areas required for cannulation without the need for cardiac manipulation often no tolerated in these severe patients, and therefore implantation without ECC (source of inflammation and coagulopathy) Becomes possible. In the series of Bantayehu Sileshi et al. [15] including 51 implanted HeartWare patients in transplantation, including 18 minimally invasive patients without ECC. The choice of the approach was made by a multidisciplinary committee, taking into account the contraindications particularly respiratory thoracotomy and the length of the ascending aorta thus the permeability of the venous bypass in case of myocardial revascularization previous. Univariate analysis revealed a statistically significant reduction of days with inotropic drugs $(p=0.04)$, and peroperative blood administration $(\mathrm{p}=0.08)$ in patients undergoing minimally invasive surgery. There was no difference in length of stay in intensive care $(\mathrm{p}=0.5)$, total length of stay $(p=0.76)$, postoperative administration of blood products $(p=0.34)$ and the total mechanical ventilation time $(\mathrm{p}=0.32)$. There were 4 intrahospital deaths, all of which were operated by sternotomy under ECC and 4 patients had a stroke, 3 of which were operated by the classical approach. The authors also show the disadvantages of sternotomy such as increased risk of infection, bleeding and complications of redo-sternotomy at the time of transplantation. In addition, the opening of the pericardium in these patients may be associated with an increased risk of right ventricular (RV) dilation and altered RV pressure-volume relationship [18].

Less invasive surgical approaches have been developed with the hope of reducing ECC time, minimizing perioperative blood loss and preserving the geometry of Heart [19].

Haberl et al. recently described their clinical experience in minimally invasive implantation for HeartWare and HeartMate II [20]. Of the 27 patients in their study, $5(19 \%)$ were performed without ECC. They had an intrahospital mortality of $14.8 \%$, and an average hospital stay of 30 days. They concluded that the minimally invasive implantation of LVAD is feasible and safe. Moreover, Anelechi [3] believes that this technique is inadequate for patients with history of cardiac surgery without giving any arguments.

In our patient who was operated twice for myocardial revascularization; a minimally 
invasive approach was been chosen. This choice was based on the surgical difficulty with increased risks by median sternotomy. Its risks mainly consist in the possibility of accidental grafting wounds which are still permeable therefore the risk of haemorrhage and myocardial ischemia in a patient with severe heart failure.

The use of this approach was also recommended for explantation or the change of the assistance slide [21-25]. For example the team of Igor [21] showed the superiority of the costal route alone in the change of HeartMate XVE by HeratMate II compared to a sternotomy associated with the left costal pathway in terms of transfusion, operative time and postoperative stay with a significant difference. The same results were found in the study of John $\mathrm{m}$. Stulak and colleagues [24].

In our patient the change of device was early (D2 post-operative) and urgent; because of a suspicion of a thrombosis of the pump. The surgical procedure was not difficult and the immediate evolution was simple.

The complications associated with the various systems of assistance are numerous: Infections (32-45\%), bleeding (27\%), Arrhythmias (24\%); Thromboembolism and stroke (10$39 \%$ depending on the model), Polyorganic insufficiency $(20 \%)$, Systemic inflammatory syndrome and Hemolysis [9,26,27].

Infection predominates, as $41 \%$ of deaths are related to sepsis [28]. Continuous flow turbine systems have a much lower rate of complications than pulsated systems [29].

Continuous flow systems have essentially three disadvantages:

- A malfunction induces the equivalent of acute aortic insufficiency because there is no valve in the system.

- Since they generate negative pressure in the LV, there is a risk of gas embolism by aspiration of air at the sutures;

- Continuous flow causes stasis at the aortic valve; this can give rise to thrombus, with the risk of systemic embolism.

This last complication interests us specifically, because we think it was the most serious complication in our patient. She caused a stroke with neurological repercussions and thrombosis of the pump with the need to change it urgently with all the complications that followed such as bleeding and infection (mediastinitis).
Ventricular assist device (VAD) thrombosis is associated with significant morbidity and mortality, usually requiring replacement of the device. Since 2011, there has been a significant increase in the incidence of VAD thrombosis from $2.2 \%$ before 2011 to $8.4 \%$ in 2013 [30]. The exact reason for this increase is unknown [31]. Diagnostic markers, including increased plasma lactate dehydrogenase (LDH), free plasma hemoglobin, or abnormal responses to programmed increases in pump speed (ramp test) [32] should allow for early and more accurate diagnosis [33].

The formation of thrombus in the aortic root in patients implanted with HeartMate II has been reported previously in the literature [34,35]. The flow in the aorta root in patients with continuous flow LVAD has been experimentally demonstrated to be relatively stasing, especially when the aortic valve does not open [36] and such stasis often involves the non-coronary sinus and may be an important risk factor for thrombosis. Sachin Shah and colleagues report a case of left coronary occlusion by an aortic root thrombus in a patient with a HeartMate II [37].

The optimal strategy for the prevention of this complication is not yet well defined; however, particular attention to anticoagulation and anti-platelet therapy in the post-operative period, as well as pump speed adjustment to allow intermittent opening of the aortic valve may be important. For those who develop an aortic root thrombus but remain asymptomatic, the intensification of anticoagulation and antiplatelet therapy alone may sometimes be sufficient [37]. In our case, management of anticoagulation and post-operative bleeding may not be optimal with formation of a thrombus in the aortic root after surgery.

Despite the technical progress of the material and the encouraging results of the implantation of artificial hearts in particular by the minimally invasive approach, it remains a heavy surgery with sometimes lethal complications. For this, the indications must be well considered by a multidisciplinary team taking into consideration some limits such as the age of the patient and his medical history.

\section{Conclusion}

Given the limited number of organ donors, long-term ventricular assist systems are gradually becoming an alternative to cardiac transplantation. Significant technological advances have resulted in the development of 
small, space-saving ventricular assist devices with fewer complications.

Alternative minimally invasive approaches for implantation or explantation of left ventricular assist devices have become valid and reproducible. Nevertheless, the complications in particular thrombo-embolic remain always serious. Only a multidisciplinary work combining surgeon, cardiologist, anesthetistresuscitator and nurse with perfect knowledge of the management of these patients and these machines prevent a sometimes fatal evolution.

At present, other technical advances and other studies are needed for the prevention and optimal management of these assistances and their potential complications.

\section{Acknowledgements}

This manuscript has not been published previously and that it is not under consideration for publication elsewhere.

All authors are in agreement with the content of the manuscript.

\section{Declaration}

No conflicts of interest. 


\section{REFERENCES}

Terracciano CM, Miller LW, Yacoub MH. Contemporary use of ventricular assist devices. Annu. Rev. Med. 61, 255-270 (2010).

Vegas A. Assisting the failing heart. Anesthesiol. Clin. 26, 539-564 (2008).

Anelechi CA. Nonsternotomy Approaches to Left Ventricular Assist Device placement: Combined Left SubcostalRight Minithoracotomy Technique. Oper. Tech. Thorac. Cardiovasc. Surg. 19(3), 254-275 (2014).

Flecher E, Joudinaud T, Grinda JM. Histoire de l'assistance circulatoire mécanique et du cour artificiel. Ann. chir. 131(8), 473-478 (2006).

Christiansen S, Klocke A, Autschbach R. Past, present, and future of long term mechanical cardiac support in adults. J. Card. Surg. 23, 664-676 (2008).

Badiwala MV, Rao V. Left ventricular device as destination therapy: are we there yet? Curr. Opin. Cardiol. 24, 184-189 (2009).

Birks EJ, George RS, Hedger $\mathrm{M}$, et al. Reversal of heart failure with a continuous-flow left ventricular assistance device and pharmacological therapy. Circulation 123, 381-390 (2011).

Long JW, Kfoury AG, Slaughter MS, et al. Long term destination therapy with the HeartMate XVE left ventricular assist device: improved outcomes since the REMATCH study. Congest. Heart Fail.11, 133-138 (2005).

Rose EA, Gelijns AC, Moskowitz AJ, et al. REMATCH study group. Long-term mechanical left ventricular assistance for end-stage heart failure. N. Engl. J. Med. 345, 1435-1443 (2001).

El-Magharbel I. Ventricular assist devices and anesthesia. Semin. Cardiothor. Vasc. Anesth. 9, 241-249 (2005).

Mohacsi P, Stalder M, Martinelli M, et al. Transplantation cardiaque et assistance circulatoire mécanique. Situation actuelle et perspectives. 2ème partie. Forum Med. Suisse 11, 98-102 (2011).
Thunberg CA, Gaitan BD, Arabia FA, et al. Ventricular assist devices today and tomorrow. J. Cardiothorac. Vasc. Anesth. 24, 656-680 (2010).

Frazier OH, Gemmato C, Myers TJ, et al. Initial clinical experience with the HeartMate II axial-flow left ventricular assist device. Tex. Heart Inst. J.34, 275281 (2007).

Chad EW, Julien SB, Jason $\mathrm{K}$, et al. Minimally invasive thoracic left ventricular assist device implantation: Case series demonstrating an integrate multidisciplinary strategy. $J$. Cardiothorac. Vasc. 29(2), 271-274 (2015).

Bantayehu S, Nicholas AH, Mary ED, et al. In-hospital outcomes of minimally invasive off-pump left thoracotomy approach using a centrifugal continuous-flow left ventricular assist device. J. Heartlung. Transplant. 34, 107-112 (2015).

Anson C, Yoan L, Annemarie K, et al. Offpump implantation of the HeratWare HVAD left ventricular assist device through minimally invasive incisions. Ann. Thorac. Surg. 91, 1294-1296 (2011).

Schmitto JD, Molitoris U, Haverich A, et al. Implantation of a centrifugal pump as a left ventricular assist device through a novel, minimized approach: upper hemisternotomy combined with antero-lateral thoracotomy. J. Thorac. Cardiovasc. Surg. 143, 511-513 (2012).

Unsworth B, Casula RP, Kyriacou AA, et al. The right ventricular annular velocity reduction caused by coronary artery bypass graft surgery occurs at the moment of pericardial incision. Am. Heart J.159, 314-322 (2010).

Schmitto JD, Mokashi SA, Cohn LH. Minimally-invasive valve surgery. J. Am. Coll. Cardiol. 56, 455-462 (2010).

Haberl T, Riebandt J, Mahr S, et al. Viennese approach to minimize the invasiveness of ventricular assist device implantation. Eur. J. Cardiothorac. Surg. (in press).

Igor D, Brian A, Leon J, et al. Clinical Experience With Sternotomy
VersusSubcostal Approach for Exchange of the HeartMateXVE to the HeartMate II Ventricular Assist Device. Ann. Thorac. Surg.85, 16461650 (2008).

Sajjad M, Butt T, Oezalp F, et al. An alternative approach to explantation and exchange of theHeartWare left ventricular assist device. Eur. J. Cardiothorac. Surg. 43, 1247-1250 (2013).

Diana GS, Prashant NM, Bartlomiej Z, et al. Minimally invasive access for off-pump HeartWare left ventricular assist device explantation. Interact. Cardiovasc. Thorac. Surg.17, 581-582 (2013).

John MS, Jennifer C, Jonathon WH, et al. Device exchange after primary left ventricular assist device implantation: indications and outcomes. Ann. Thorac. Surg.95, 1262-1268 (2013).

Rojas SV, Avsar M, Khalpey Z, et al. Minimally invasive pff_pump left ventricular assist device exchange: anterolateral thoracotomy. Artif. Organs 38(7), 539-542 (2014).

Deng MC, Edwards LB, Herrtz MI, et al. Mechanical circulatory support data base of the international society for heart and lung transplantation: second annual report 2004. J. Heart Lung Transplant. 23, 1027-1034 (2004).

Stone ME. Current status of mechanical circulatory assistance. Semin. Cardiothor. Vasc. Anesth. 11, 185-204 (2007).

Christiansen S, Klocke A, Autschbach R. Past, present, and future of long term mechanical cardiac support in adults. J. Card. Surg. 23, 664-676 (2008).

Mitter N, Sheinberg R. Update on ventricular assist devices. Curr. Opin. Anaesthesiol. 23, 57-66 (2009).

Starling RC, Moazami N, Silvestry SC, et al. Unexpected abrupt increase in left ventricular assist device thrombosis. N. Engl. J. Med. 370, 33-40 (2014).

Edo Y, Birati, Rame E. Diagnosis and Management of LVAD Thrombosis. 
Curr. Treat. Options Cardio. Med.17, 2 (2015).

Uriel N, Morrison KA, Garan AR, et al. Development of a novel echocardiography ramp test for speed optimization and diagnosis of device thrombosis in continuous-flow left ventricular assist devices: the Columbia Ramp Study. J. Am. Coll. Cardiol. 60, 1764-1765 (2012).

Uriel N, Han J, Morrison KA, et al.Device thrombosis in HeartMate II continuous-flow left ventricular assist devices: a multifactorial phenomenon. J. Heart Lung Transplant 33, 51-9 (2014).

Freed BH, Jeevanandam V, Jolly N. Aortic root and valve thrombosis after implantation of a left ventricular assist device. J. Invasive Cardiol. 23, 63-65 (2011).

Demirozu Z, Frazier O. Aortic valve noncoronary cusp thrombosis after implantation of a nonpulsatile, continuous-flow pump. Tex. Heart Inst. J. 39(5), 618-620 (2012).
Kar B, Delgado R, Frazier O, et al. The effect of LVAD aortic outflow-graft placement on hemodynamics and flow. Implantation technique and computer flow modeling. Tex. Heart Inst. J. 32, 294-298 (2005).

Sachin S, Mehra M, Couper G, Desa A. Continuous flow left ventricular assist device related aortic root thrombosis complicated by left main coronary artery occlusion. J. Heart Lung Transplant. 33(1), 119-120 (2014). 